\title{
Canonical Wnt inhibitors ameliorate cystogenesis in a mouse ortholog of human ADPIKD
}

\author{
Ao Li, ${ }^{1,2}$ Yuchen Xu, ${ }^{1}$ Song Fan, ${ }^{1}$ Jialin Meng, ${ }^{1}$ Xufeng Shen, ${ }^{1}$ Qian Xiao, ${ }^{2}$ Yuan Li, ${ }^{3}$ Li Zhang, \\ Xiansheng Zhang, ${ }^{1}$ Guanqing $\mathbf{W u},{ }^{1,3}$ Chaozhao Liang, ${ }^{1}$ and Dianqing $\mathbf{W u}^{2}$ \\ ${ }^{1}$ Anhui Province PKD Center, Institute and Department of Urology, The First Affiliated Hospital of Anhui Medical \\ University, Hefei, Anhui Province, China. ${ }^{2}$ Department of Pharmacology, Yale University School of Medicine, New Haven, \\ Connecticut, USA. ${ }^{3}$ State Key Laboratory of Molecular Oncology, Cancer Hospital and Institute, Chinese Academy \\ of Medical Sciences and Peking Union Medical College, Beijing, China.
}

Autosomal dominant polycystic kidney disease (ADPKD) can be caused by mutations in the PKD1 or PKD2 genes. The PKD1 gene product is a Wnt cell-surface receptor. We previously showed that a lack of the PKD2 gene product, PC2, increases $\beta$-catenin signaling in mouse embryonic fibroblasts, kidney renal epithelia, and isolated renal collecting duct cells. However, it remains unclear whether $\beta$-catenin signaling plays a role in polycystic kidney disease phenotypes or if a Wnt inhibitor can halt cyst formation in ADPKD disease models. Here, using genetic and pharmacologic approaches, we demonstrated that the elevated $\beta$-catenin signaling caused by PC2 deficiency contributes significantly to disease phenotypes in a mouse ortholog of human ADPKD. Pharmacologically inhibiting $\beta$-catenin stability or the production of mature Wnt protein, or genetically reducing the expression of Ctnnb1 (which encodes $\beta$-catenin), suppressed the formation of renal cysts, improved renal function, and extended survival in ADPKD mice. Our study clearly demonstrates the importance of $\beta$-catenin signaling in disease phenotypes associated with Pkd2 mutation. It also describes the effects of two Wnt inhibitors, XAV939 and LCK974, on various Wnt signaling targets as a potential therapeutic modality for ADPKD, for which there is currently no effective therapy.

Conflict of interest: The authors have declared that no conflict of interest exists.

License: This work is licensed under the Creative Commons Attribution 4.0 International License. To view a copy of this license, visit http:// creativecommons.org/licenses/by/4.0/

Submitted: June 26, 2017

Accepted: January 23, 2018

Published: February 22, 2018

Reference information:

JCI Insight. 2018;3(4):e95874. https:// doi.org/10.1172/jci.insight.95874.

\section{Introduction}

Autosomal dominant polycystic kidney disease (ADPKD) is the most common of a group of inherited kidney disorders characterized by progressive cyst development and various extrarenal manifestations ( 1 , 2). Cystogenesis in the human kidneys can progressively occupy the normal parenchyma of the kidney and lead to renal failure, which usually occurs in mid-to-late adulthood. This disease is the fourth most common single cause of end-stage renal failure worldwide $(3,4)$.

ADPKD is caused by mutations in the $P K D 1$ or $P K D 2$ genes, which encode the proteins polycystin-1 (PC1) and polycystin-2 (PC2), respectively. Approximately 85\% of ADPKD patients have mutations in $P K D 1$, and the remaining $15 \%$ have mutations in $P K D 2(5,6)$. The most common extrarenal manifestation of ADPKD is the formation of bile duct-derived cysts in the liver $(2,7)$. Liver cysts occur in $83 \%$ of all ADPKD patients, and $94 \%$ of the patients with liver cysts are over 35 years old $(8,9)$. Other ADPKD phenotypes include pancreatic cysts $(10,11)$, aneurysms $(12-15)$, and aortic root/thoracic aorta abnormalities (16-18).

There has been considerable progress in elucidating the molecular mechanisms and pathogenesis of $\operatorname{ADPKD}(3,5,19)$. Recent studies show that human cystic disease may involve Wnt signal transduction (20-22). Wnt signaling is a highly conserved molecular pathway that regulates cell fate and embryogenesis/ organogenesis and homeostasis in vertebrates. Intracellular Wnt signaling can be classified into canonical and noncanonical pathways. Both Wnt signaling pathways have been proposed to have a link to ADPKD progression in animal models and human patients (20, 21, 23-25). Hitherto, many reports have shown that renal cystic disease may result from dysregulation of the noncanonical Wnt pathway by disrupting Wnt/ $\mathrm{Ca}^{2+}$ signaling and/or PCP processes in renal epithelial cells $(23,26-32)$. The roles of canonical Wnt signaling in pathogenesis of $\mathrm{ADPKD}$ remain to be unequivocally defined. A transgenic mouse for $\beta$-catenin, a key 
factor for canonical Wnt signaling, exhibits severe PKD phenotypes, indicating that $\beta$-catenin upregulation alone is sufficient to induce cyst formation in the kidney (33). Disruption of $A p c$, which is a cofactor for the $\beta$-catenin degradation complex, and overexpression of $c-M y c$, a canonical Wnt targeting product, also induce cystogenesis in the kidney of mouse models $(34,35)$. Moreover, aberrant activation of canonical Wnt signaling was detected in the spectrum of PC1- and PC2-deficient cells and tissues (29, 30, 36-38). These findings directly or indirectly support that hyperactivation of canonical Wnt signaling may cause cyst formation in the kidney of animal models. However, other reports indicate that increased canonical Wnt signaling may not play a key role in cystogenesis of $\operatorname{PKD}(25,28,31,39-41)$. The opposite has been found, as cyst formation might disturb Wnt/PCP homeostasis through lose of balance between canonical and noncanonical Wnt activity $(20,24,29,30,42,43)$. Thus far, no studies have been performed to investigate the effects of canonical Wnt signaling inhibition on cytogenesis in mouse PKD models.

Several studies have reported that many promising drugs showed potential therapeutic effects for ADP$\mathrm{KD}$ in a large number of preclinical and clinical trials $(1,7,44-54)$. However, some of those in clinical trials - these promising drugs included mTOR inhibitors $(55,56)$, somatostatin analogs $(57,58)$, ACEI/ $\mathrm{ARB}(59,60)$, and tyrosine kinase inhibitor (61) - did not prevent the decline of EGFR in ADPKD patients. Only tolvaptan, a vasopressin V2 receptor inhibitor, currently has been shown to both reduce the formation of renal cysts and improve renal function $(54,62)$, but the impairment of liver function and other side effects have been challenged to be widely used in clinic $(63,64)$. Thus, there is still an urgent need for new drugs that can suppress cystogenesis in ADPKD patients. We considered a spectrum of Wnt inhibitors with various molecular targets along the Wnt signaling pathway and identified XAV939 and LGK974 as agents that effectively reduced cystogenesis, improved renal function, and extended survival in our ADPKD animal model. Our study provides clear evidence for the importance of $\beta$-catenin signaling in $P k d 2$ mutant-associated disease phenotypes and describes the effects of the Wnt inhibitors XAV939 and LGK974 on various Wnt signaling targets. These Wnt inhibitors are potential therapeutic modalities for $\mathrm{ADPKD}$, for which there is currently no effective therapy.

\section{Results}

Reducing $\beta$-catenin, a key factor in canonical Wht signaling, delays cyst formation in a mouse model of human $A D P K D$. We previously generated an epithelial cell-specific $P k d 2$-knockout mouse line ( $V i l^{\mathrm{Cre}} P k d 2^{\mathrm{f} / \mathrm{f}}$ mice, also know as Vil-Cre; $P k d 2^{\mathrm{f} 3 / \mathrm{fs}}$ mice) by crossing Villin-Cre and $P k d 2$-floxed mice $(37,65)$. These $P k d 2$ mutant mice start developing renal cysts before 1 month of age and have an average life span of 4 months (65). The renal tissues in $\mathrm{Vil}^{\mathrm{Cre}} P k d 2^{\mathrm{f} / \mathrm{f}}$ mice have elevated levels of active, nuclear, and total $\beta$-catenin (Ctnnb1) proteins (Figure 1, A and B) and Axin2, c-Myc, and cyclin D1 proteins, which are produced by Wnt target genes (Figure 1, C and D), compared with control mice $\left(P k d 2^{\mathrm{f} / \mathrm{f}}\right)$. These results are consistent with previous reports that PC2 deficiency increases Wnt/ $\beta$-catenin signaling $(27,37,66)$. To determine the importance of $\beta$-catenin in disease phenotypes in the $V i l^{\mathrm{Cre}} P k d 2^{\mathrm{f} / \mathrm{f}}$ mice, we crossed $\mathrm{Vil} \mathrm{Cre}^{\mathrm{Cre}} \mathrm{Pkd} 2^{\mathrm{f} / \mathrm{f}}$ mice (65) with $C t n n b 1^{+/-}$mice to generate $V i l^{\mathrm{Cre}} P k d 2^{\mathrm{f} / \mathrm{f}} \mathrm{Ctnnb1}^{+/-}$mice (Figure 2A). The loss of one Ctnnb1 allele rescued the elevated levels of active, nuclear, and total $\beta$-catenin found in the kidneys of $V i l^{\mathrm{Cr}} P k d 2^{\mathrm{f} / \mathrm{f}}$ mice, bringing the levels down almost to those of control $P k d 2^{\mathrm{f} / \mathrm{f}}$ mice, which did not have Vil-Cre (Figure 1, A and B). The loss of one Ctnnb1 allele also reduced the elevated levels of Axin2, c-Myc, and cyclin D1 back to control levels (Figure 1, C and D). Kaplan-Meier survival analysis showed that $V i l^{\mathrm{Cre}} P k^{\mathrm{C} d} 2^{\mathrm{f}}$ ${ }_{\mathrm{f}}^{\mathrm{f}} \mathrm{tnnb} 1^{+/-}$mice had a significantly longer life span than $\mathrm{Vil}{ }^{\mathrm{Cre}} P k d 2^{\mathrm{f} / \mathrm{f}}$ mice (Figure $2 \mathrm{~B}$ ). Histology revealed

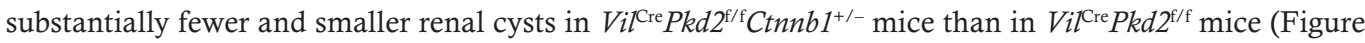

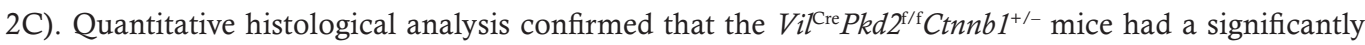
lower renal cystic index than $\mathrm{Vil}^{\mathrm{Cre}} \mathrm{Pkd} 2^{\mathrm{f} / \mathrm{f}}$ mice (Figure 2D). The $\mathrm{Vil} \mathrm{Cre}^{\mathrm{Ce}} \mathrm{Pkd} 2^{\mathrm{f} / \mathrm{f}} \mathrm{Ctnnb1^{+/- }}$ mice also had significantly improved kidney/body weight ratio (Figure 2E), renal function parameters (Figure 2, F and $\mathrm{G}$ ), and renal fibrosis (Supplemental Figure 1A; supplemental material available online with this article; https://doi.org/10.1172/jci.insight.95874DS1) compared with $V i l^{\mathrm{Cre}} P k d 2^{\mathrm{f} / \mathrm{f}}$ mice. Together, these results indicated that elevated $\beta$-catenin levels, due to the loss-of-function $P k d 2$ mutation, contribute to the disease phenotype. Of note, we did not observe any differences in morphology or renal function parameters between $\mathrm{Vil}^{\mathrm{Cre}} \mathrm{Ctnnb1}^{+/-}$mice and their control littermates (data not shown).

$\mathrm{Wnt} / \beta$-catenin signaling is implicated in the regulation of proliferation and apoptosis (67-70). Examination of the cyst-lining epithelial cells by cleaved caspase- 3 and TUNEL staining revealed that the loss of a $\beta$-catenin allele did not alter apoptosis (Figure 2, H and I, and Supplemental Figure 1B). 


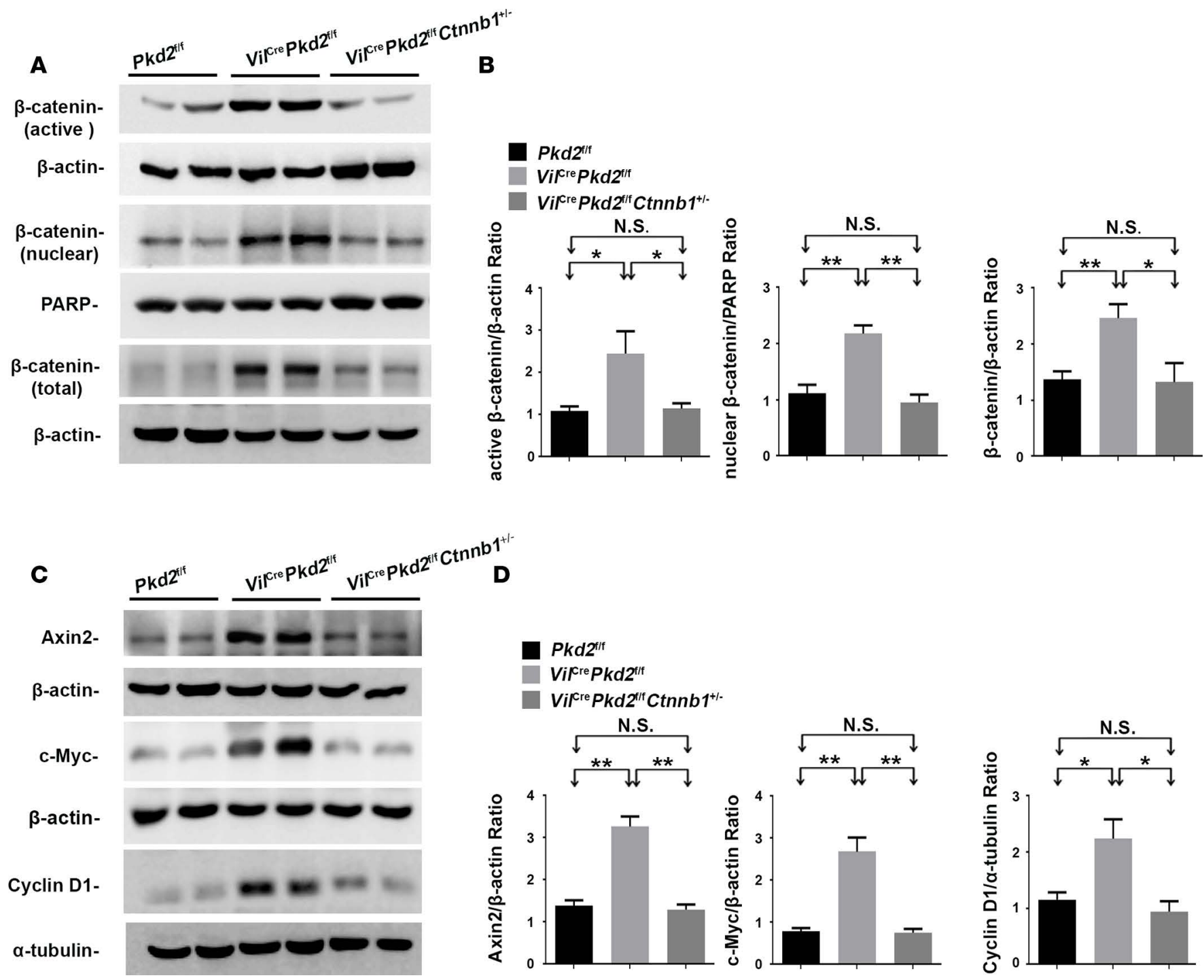

Figure 1. Effects of allelic reduction of the Ctnnb1 gene. (A and B) Allelic reduction of the Ctnnb1 gene reduced the active, nuclear, and total $\beta$-catenin

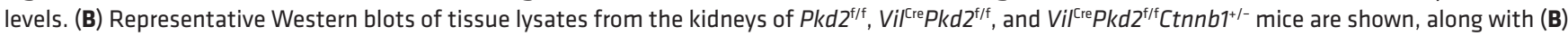
normalized quantitative analysis of the densitometry values of the tested tissues. (C and $\mathbf{D})$ Allelic reduction of the $C$ tnnb1 gene suppressed $\beta$-cateninmediated transcription (including Axin2, c-Myc, and cyclin D1) activated by PC2 deficiency. All data are presented as mean $\pm \mathrm{SEM}\left({ }^{*} P<0.05,{ }^{* *} P<0.01\right.$, Student's $t$ test). Data are from 3 animals/group.

However, the loss of a Ctnnb1 allele rescued the elevated proliferation of cyst-lining renal epithelial cells seen in $\mathrm{Vil}^{\mathrm{Cre}} \mathrm{Pkd} \mathrm{f}^{\mathrm{f} / \mathrm{f}}$ mice, returning the proliferation almost to control levels (Figure 2, $\mathrm{J}$ and $\mathrm{K}$ ). These results suggested that elevated $\beta$-catenin may be responsible for increasing the renal epithelial cell proliferation in $\mathrm{Vil}^{\mathrm{Cr}} \mathrm{Pkd2^{ \textrm {f } / \mathrm { f } }}$ mice.

XAV939, which inhibits canonical Wnt signaling, impedes cystogenesis in Pkd2 mutant kidneys. XAV939 is a tankyrase inhibitor. It antagonizes Wnt signaling by stabilizing Axin proteins, thereby accelerating $\beta$-catenin degradation (71). In $V_{i}{ }^{\mathrm{Cre}} P k d 2^{\mathrm{ff}}$ mice, XAV939 treatment $(50 \mathrm{mg} / \mathrm{kg}$ i.p. daily, Figure $3 \mathrm{~A}$ ) starting at $\mathrm{P} 10$ reduced the $\beta$-catenin and Wnt target gene products to the levels in control $P k d 2^{f / f}$ mice (Supplemental Figure 2, A-D). Consistent with its effects on $\beta$-catenin signaling, XAV939 treatment significantly impeded the progression of renal cystogenesis (Figure 3, B and C), decreased the kidney/body weight ratio (Figure 3D), and improved renal function parameters (Figure 3, $\mathrm{E}$ and $\mathrm{F}$ ) in $\mathrm{Vil}^{\mathrm{C} r e} P k d 2^{\mathrm{f} / \mathrm{f}}$ mice. Moreover, XAV939 treatment reduced the number of proliferating cyst-lining renal epithelial cells, without significantly affecting their apoptosis (Figure 3 , G-J). Importantly, this treatment significantly improved survival (Figure $3 \mathrm{~K}$ ). These data strongly supported the hypothesis that elevated $\beta$-catenin due to a $P k d 2$ loss-of-function mutation contributes to the disease phenotype. 
A
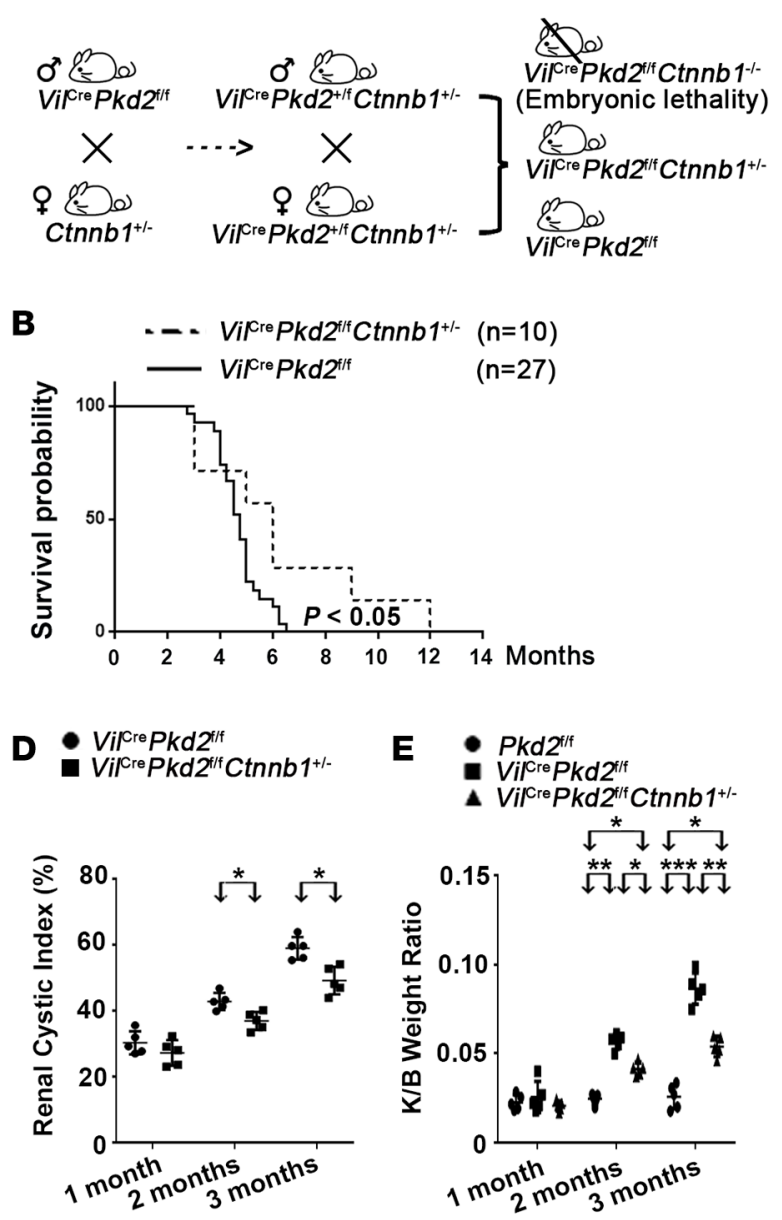

H $\square$ Pkd2/ff

Vifre $P k d 2^{\prime / f}$ VilCre Pkd2/ff Ctnnb1 ${ }^{+/-}$
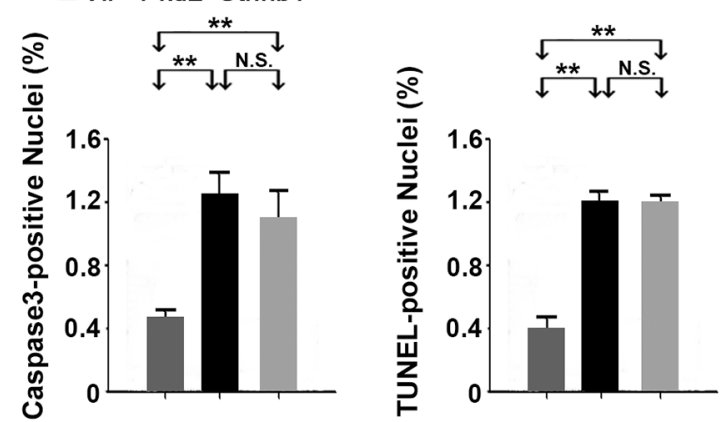

C

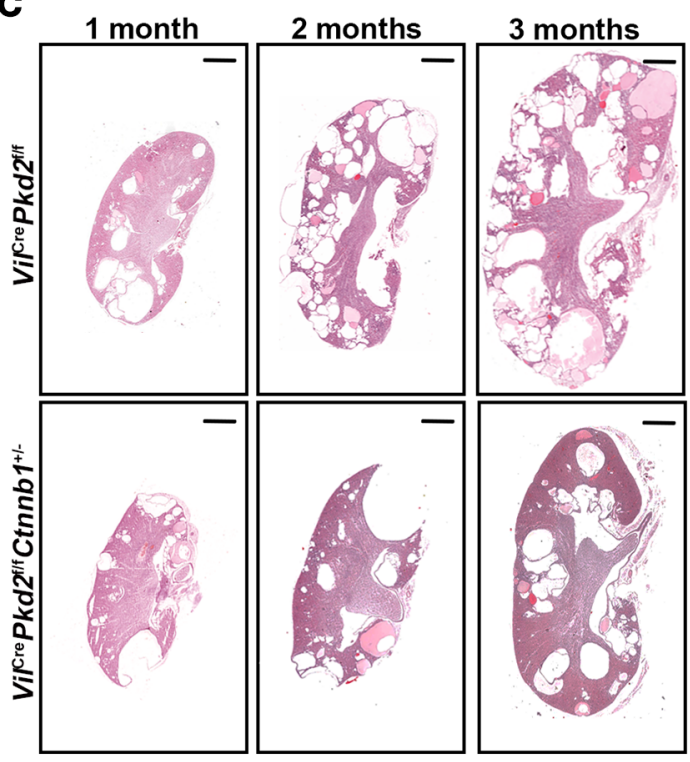

$\mathbf{F}$

G

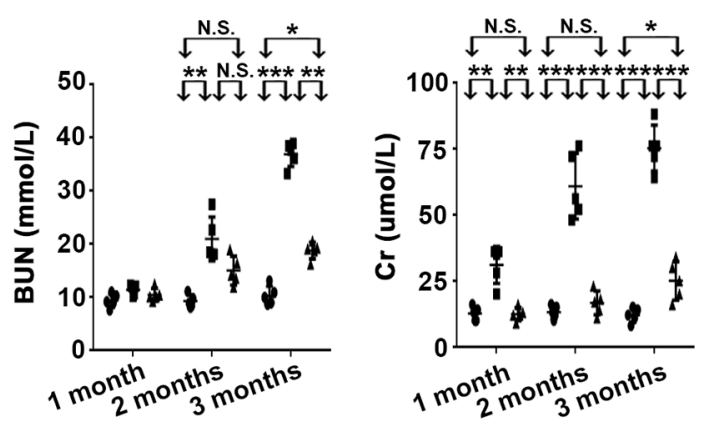

J

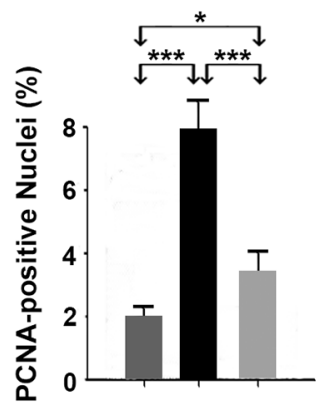

$\mathbf{K}$

$P k d 2^{\text {f/f }}$

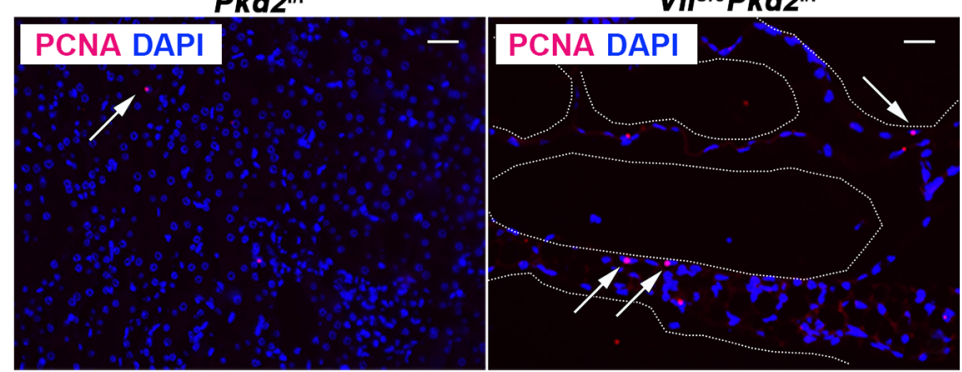

Vilcre Pkd2 ${ }^{\text {fif }}$ Ctnnb1 1/-

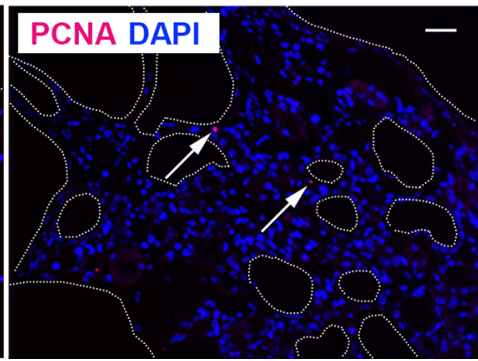




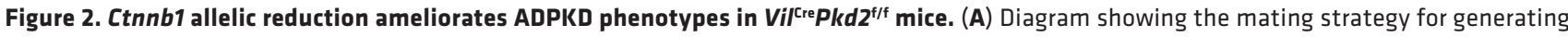

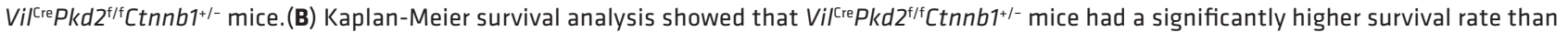
$V_{i l \mid c r e} P k d 2^{f / f}$ mice. (C) Representative histology of kidney sections from mice at 1, 2, and 3 months of age. Scale bars: $600 \mu \mathrm{m}$. (D and $\left.\mathbf{E}\right)$ Renal cystic index and kidney/body weight ratio. (F and $\mathbf{G}$ ) Renal function parameters: blood urea nitrogen (BUN) and creatinine (Cr). Data in $\mathbf{D}-\mathbf{G}$ are presented as mean \pm SD $\left({ }^{*} P<0.05,{ }^{* *} P<0.01,{ }^{* *} P<0.001, n=5\right.$, ANOVA). ( $\mathbf{H}$ and $\left.\mathbf{I}\right)$ The loss of one Ctnnb1 allele did not affect apoptosis of cyst-lining epithelial cells, as assessed by cleaved caspase-3 and TUNEL staining. (J and $\mathbf{K}$ ) The loss of one Ctnnb1 allele reduced the proliferation of cyst-lining epithelial cells, as detected by PCNA staining. Arrows indicate positive PCNA staining. Data in $\mathbf{H}$-J are presented as mean \pm SD $\left({ }^{*} P<0.05,{ }^{* *} P<0.01,{ }^{* *} P<\right.$ $0.001, n=3$, ANOVA). Scale bars: $60 \mu \mathrm{m}$.

LGK974, which inhibits another target of Wnt signaling, also delays cystogenesis in Pkd2 mutant kidneys. We further confirmed the importance of elevated Wnt signaling in cystogenesis using the Wnt biogenesis inhibitor LGK974, an orally bioavailable porcupine inhibitor that blocks a lipid modification of Wnt proteins that is essential for Wnt activity $(72,73)$. Much like the XAV939 treatment, LGK974 treatment ( $3 \mathrm{mg} / \mathrm{kg}$ daily intragastrically) starting from P30 (Figure $4 \mathrm{~A}$ ) reduced the levels of $\beta$-catenin and Wnt

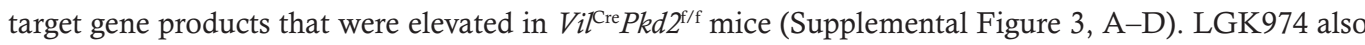
significantly impeded the progression of renal cystogenesis (Figure 4, B and C), decreased the kidney/ body weight ratio (Figure 4D), improved renal function parameters (Figure 4, E and F), and reduced the number of proliferating cyst-lining renal epithelial cells without affecting their apoptosis in $\mathrm{Vil}^{\mathrm{Cre}} P \mathrm{Pd}^{\mathrm{f} / \mathrm{f}}$

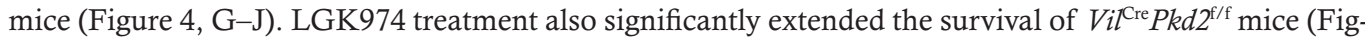
ure $4 \mathrm{~K}$ ). Thus, we obtained very similar results from a genetic Ctnnb1 allelic reduction and treatment with two distinct Wnt pathway inhibitors.

Upregulation of $W n t / \beta$-catenin signaling depends on the presence of $P C 2$. We previously established renal collecting ductal epithelial cell lines from WT and $P k d 2^{\mathrm{f} /-}$ (its genotype can be considered as $P k d 2^{+/-}$) mice and established a $P k d 2$-null cell line $\left(P k d 2^{-/-}\right.$cells) by transducing the $P k d 2^{\mathrm{f} /-}$ cells with a Cre-expressing adenovirus $(37,74)$. We observed elevated Wnt reporter gene activity and increased expression of the Wnt target gene Axin2 in $P k d 2^{-1-}$ cells compared with that in $P k d 2^{+/-}$cells (37). When these cell lines were treated with LGK974, the protein levels of active, nuclear, and total $\beta$-catenin were markedly reduced in the $P k d 2^{-/-}$cells (Supplemental Figure 4A); Axin2, c-Myc, and cyclin D1 were also reduced (Supplemental Figure 4B). XAV939 treatment produced the same results (Supplemental Figure 4, C and D). These results suggested that PC2 suppresses Wnt/ $\beta$-catenin signaling in an autonomous manner. Further supporting this conclusion, siRNA-mediated $\beta$-catenin silencing abrogated the elevated Wnt reporter gene activity in $P k d 2^{-/-}$cells (Supplemental Figure 4E).

To reveal the functional role of PC2 loss in activating Wnt/ $\beta$-catenin signaling, we analyzed gene expression by microarray analyses of WT and $P k d 2^{-/-}$cells. Confirming our earlier conclusions, we found that gene expression was significantly altered in the Wnt/ $\beta$-catenin signaling pathway (Supplemental Figure 5A). Gene expression analysis also revealed the increased gene expression of Ctnnb1 and several Wnt genes in $P k d 2^{-1-}$ cells (Supplemental Figure 5B). Quantitative RT-PCR showed that the expression of Ctnnb1, $W n t 7 a$, and $W n t 9 a$ was elevated in $P k d 2^{-/-}$cells compared with maternally derived $P k d 2^{+/-}$cells (37) (Figure 5, A-C). To further confirm that a loss of PC2 was responsible for upregulating Ctnnb1, Wnt7a, and Wnt9a, we reexpressed human $P K D 2$ in the $P k d 2^{-/-}$cells and found that expression of these genes, which was elevated in cells without PC2, returned to the levels expressed in cells with PC2 $\left(P k d 2^{+/+}\right.$or $P k d 2^{+/-}$) (Supplemental Figure 6, A-E). PKD2 reexpression also reduced the expression of the Wnt target genes Axin2, c-Myc, and $C$ cnd 1 to normal levels (Supplemental Figure 6, F-H). These results indicated that the alterations we observed in Wnt gene expression were indeed dependent on the presence of PC2.

In addition, we also tested if PC2 expression could be dysregulated by treatment of these Wnt inhibitors. Immunofluorescence analyses showed that only control mouse kidney exhibits strong posi-

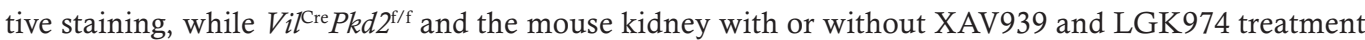
showed obvious low expression of PC2 (Supplemental Figure 7A). However, no significant differences were observed in PC2 expression among kidneys with or without LGK974 and XAV939 treatment (Supplemental Figure 7, B and C). These results indicated that treatment of these Wnt inhibitors did not alter the PC2 expression in vivo.

A lack of PC2 upregulates Wnt7a and Wnt9a expression independently of $\beta$-catenin. To further validate our findings, we treated $P k d 2^{-/-}$cells and $P k d 2$ mutant mice with the Wnt inhibitors LGK974 and XAV939. Interestingly, both Wnt inhibitors suppressed Ctnnb1 expression, but neither affected the expression of Wnt7a or Wnt $9 a$ (Figure 5, A-F, and Supplemental Figure 8, A-F). Moreover, a Ctnnb1 allelic reduction did not affect 
A

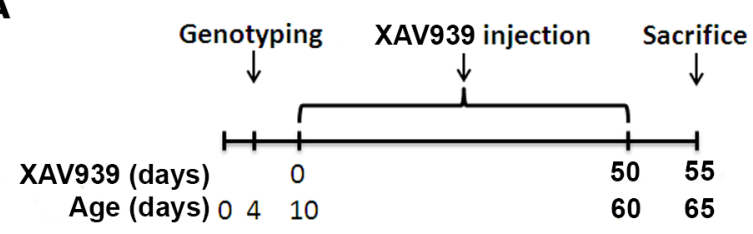

B

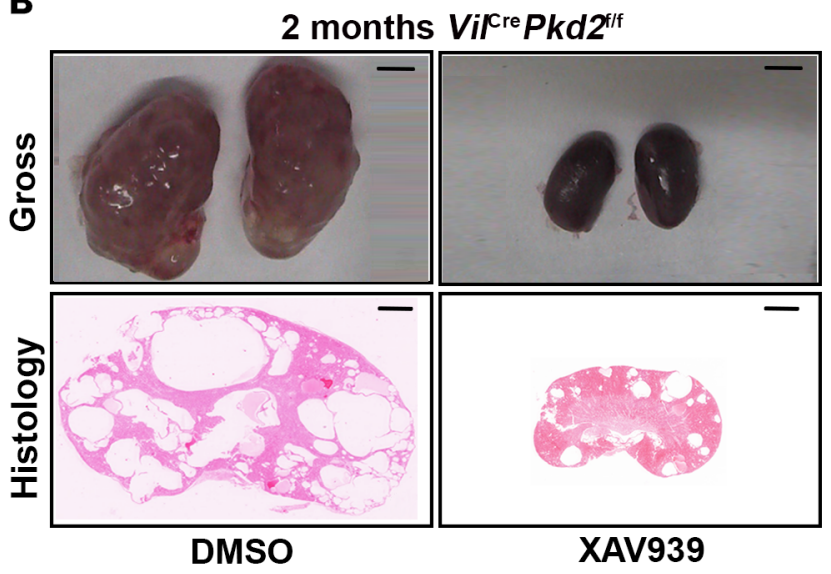

G
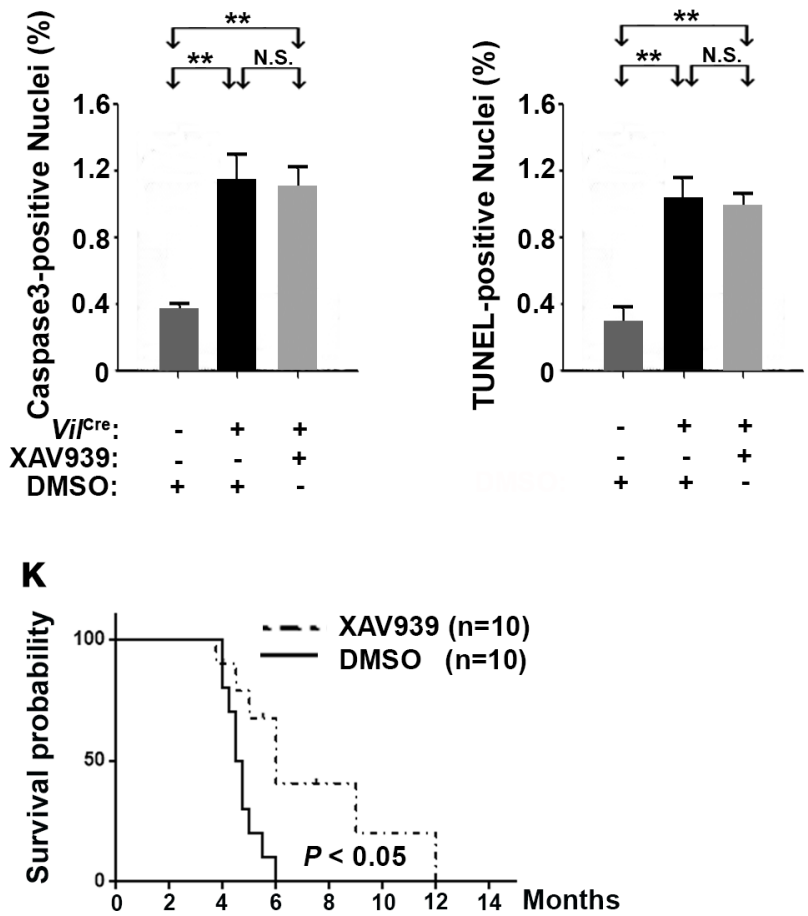
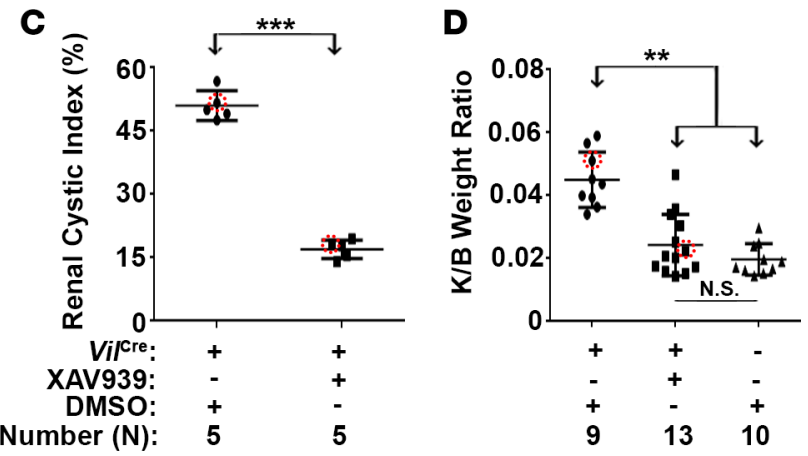

E
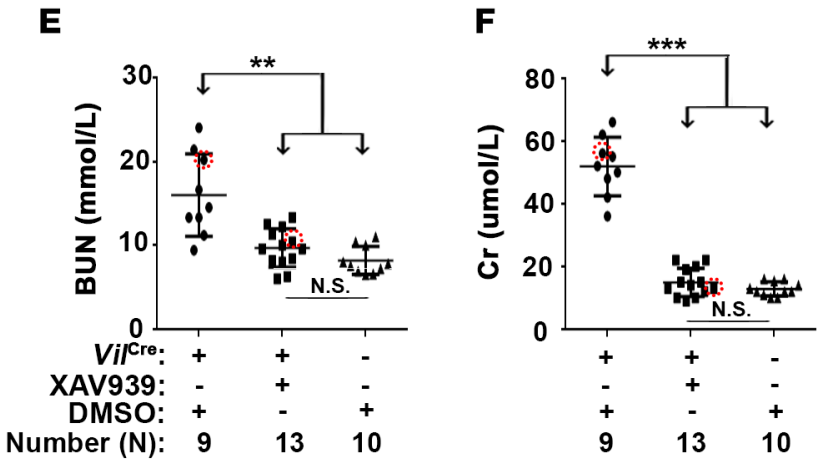

I

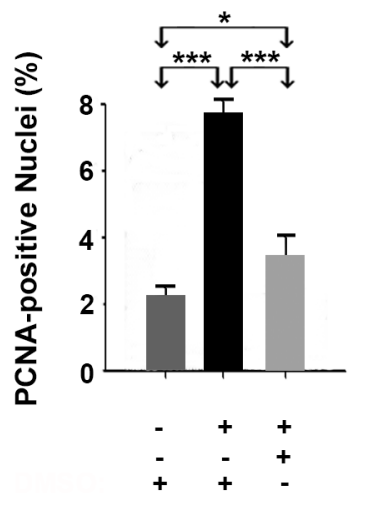

J

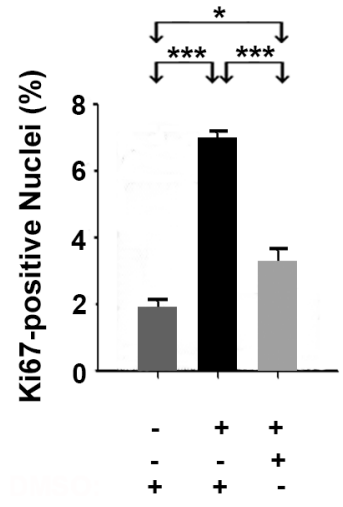

Figure 3. XAV939 impedes cyst formation in VilcrePkd2 ${ }^{\text {f/f }}$ mice. (A) Diagram showing the XAV939 injection schedule. (B) Representative kidney images and histology. Scale bars: $3 \mathrm{~mm}$ (top); $600 \mu \mathrm{m}$ (bottom). (C-F) Renal cystic index, kidney/body weight ratio, and the renal function parameters, blood urea nitrogen (BUN) and creatinine (Cr). Circled dots represent the mice from which representative kidney images and histology (B) were taken. (G and $\mathbf{H}$ ) XAV939 did not affect apoptosis but (I and J) did reduce proliferation $(n=3)$. (K) XAV939 significantly prolonged the survival of $V i / l^{\text {re }} P k d 2^{f / f}$ mice $(P<0.05)$. Data in $\mathbf{C}-\mathrm{J}$ are presented as mean \pm SD $\left({ }^{*} P<0.05 ;{ }^{* *} P<0.01,{ }^{* * *} P<0.001\right.$, ANOVA). 
A

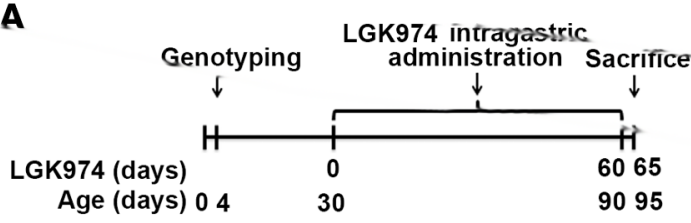

B

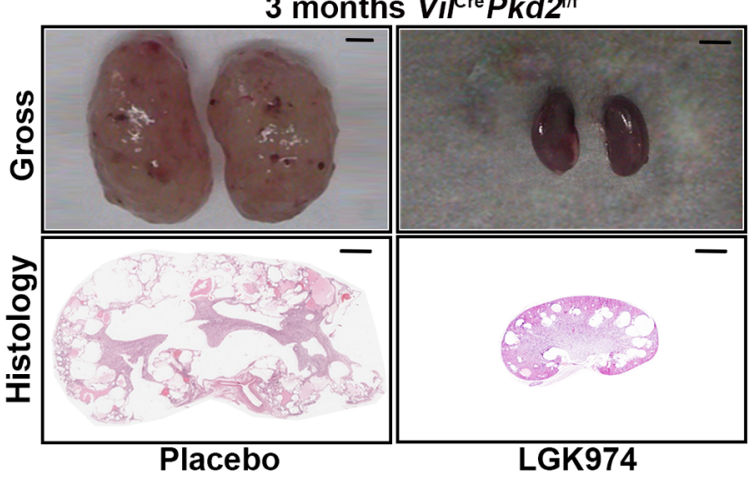

G

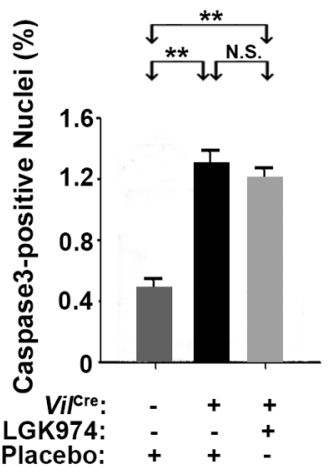

K

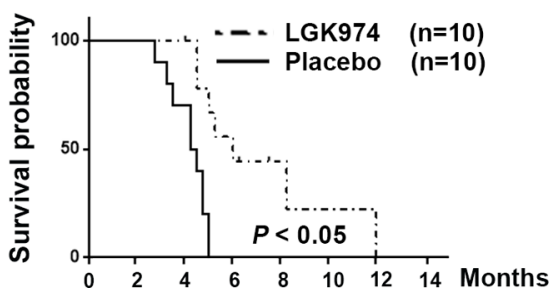

C

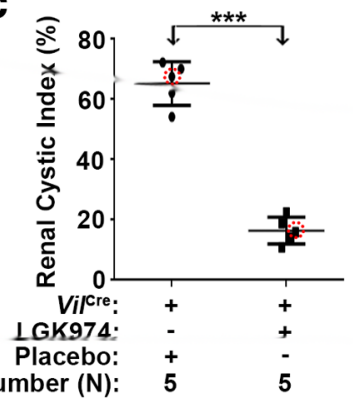

D

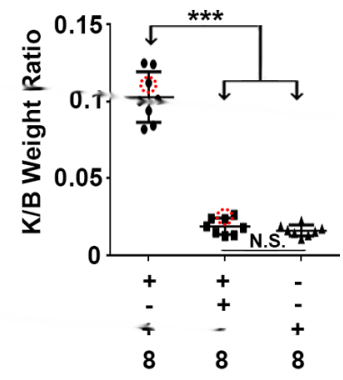

E
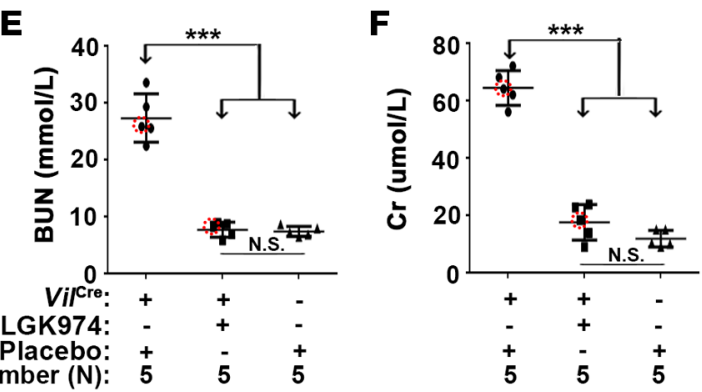

I

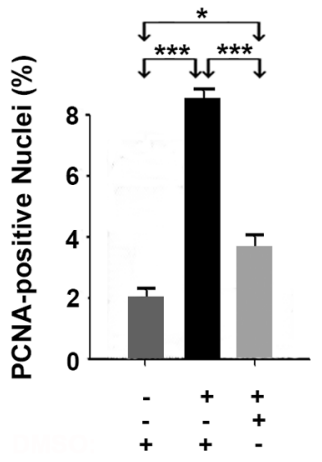

J

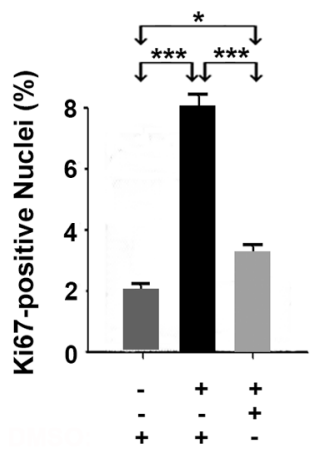

Figure 4. LGK974 treatment delayed cyst formation in VilcrePkd2 ${ }^{\text {f/f }}$ mice. (A) Diagram showing the LGK974 treatment schedule. (B) Representative kidney images and histology. Scale bars: 3 mm (top); $600 \mu \mathrm{m}$ (bottom). (C-F) Renal cystic index, kidney/body weight ratio, and the renal function parameters, blood urea nitrogen (BUN) and creatinine (Cr). Circled dots represent the mice from which representative kidney images and histology (B) were taken. (G and $\mathbf{H})$ LGK974 did not affect apoptosis but (I and J) did reduce proliferation $(n=3)$. (K) LGK974 prolonged the survival of Vilcre $P k d 2^{\mathrm{f} / \mathrm{f}}$ mice $(P<0.05)$. Data in C-J are presented as mean \pm SD $\left({ }^{*} P<0.05 ;{ }^{* *} P<0.01,{ }^{* *} P<0.001\right.$, ANOVA).

the elevation of Wnt7a or Wnt9a expression due to PC2 deficiency (Supplemental Figure 8, G-I), suggesting that the expression of $W n t 7 a$ and $W n t 9 a$ did not depend on $\beta$-catenin. We therefore investigated if inhibiting Wnt7a (Figure 5G) could alter PC2-lacking-associated $\beta$-catenin expression. Indeed, silencing Wnt7a rescued abnormally elevated $\beta$-catenin levels in $P k d 2^{-/-}$cells (Figure $5 \mathrm{H}$ ). To further confirm the result, we pretreated these cells with LGK974 and added purified Wnt7a. Results showed that exogenously purified WNT-7a 
A

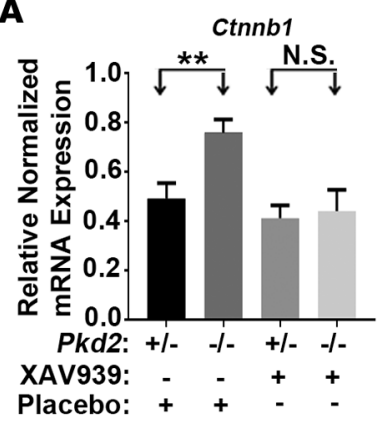

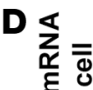

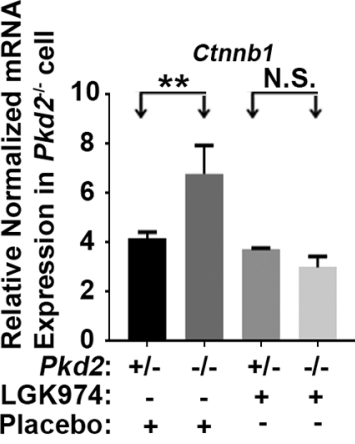

G

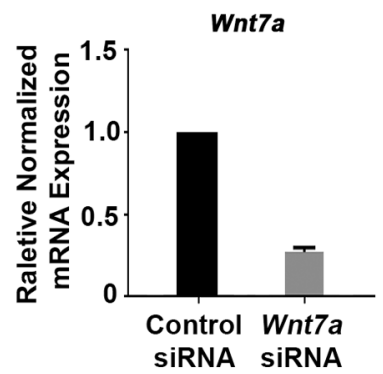

J

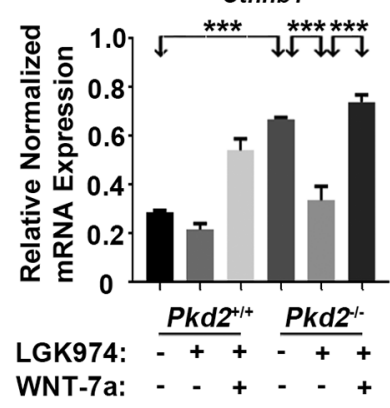

M

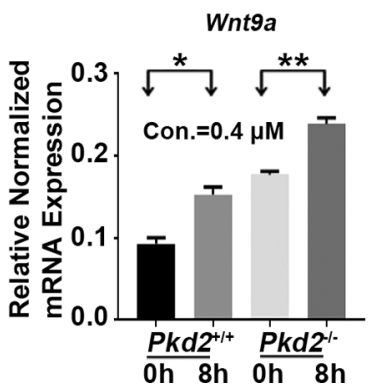

B

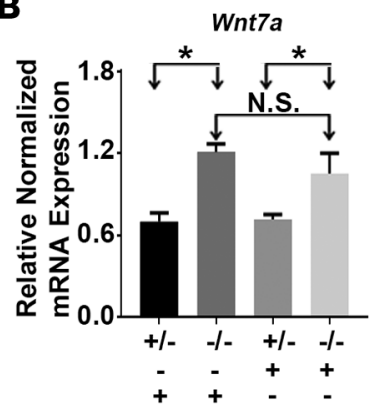

$\mathbf{E}$

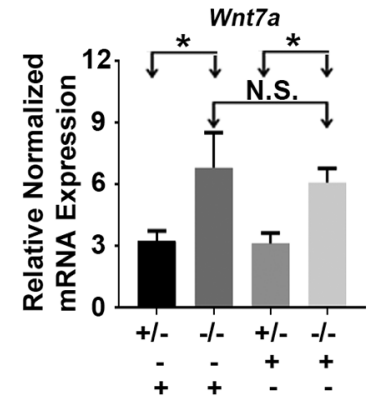

H

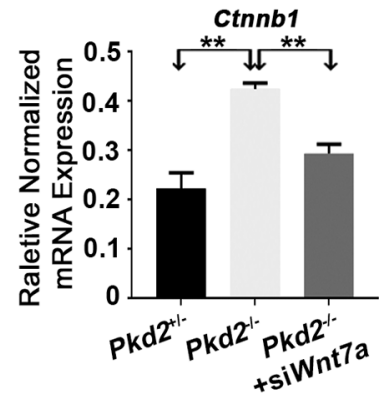

$\mathbf{K}$

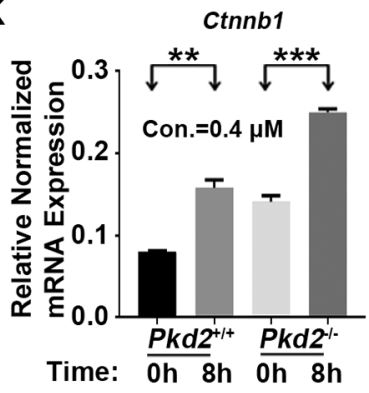

C

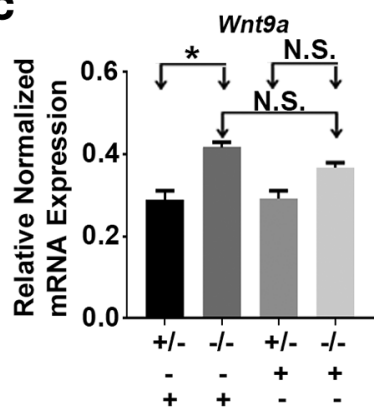

$\mathbf{F}$

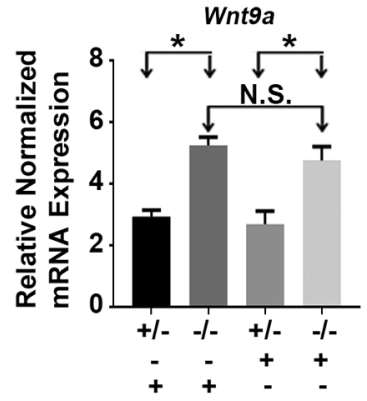

I

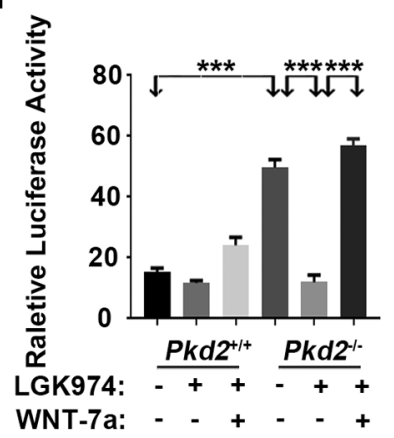

$\mathbf{L}$

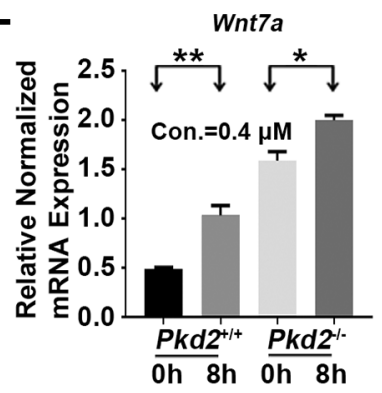

Figure 5. PC2 deficiency upregulates Ctnnb1, Wnt7a, and $\mathbf{W n t 9 a}$ expression. (A-C) XAV939 inhibited the PC2 deficiency-induced elevation of the mRNA levels of Ctnnb1 but not of Wnt7a or Wnt9a. Cells were treated with XAV939 $(2 \mu \mathrm{M})$ for 16 hours, and mRNA levels were determined by quantitative RT-PCR. (D-F) LGK974 inhibited the $P k d 2$ deficiency-induced elevation of the mRNA levels of Ctnnb1 but not of Wnt7a or Wnt9a. Cells were treated with LGK974 ( $2 \mu \mathrm{M})$ for 24 hours, and mRNA levels were determined by quantitative RT-PCR. (G) Wnt7a mRNA levels in Pkd2 ${ }^{-1-}$ cells were measured 48 hours after Wnt7a siRNA treatment by quantitative RT-PCR. (H) Wnt7a knockdown reduced the Pkd2 deficiency-induced elevation of Ctnnb1 mRNA. (I) Luciferase reporter gene activity was elevated in $P k d 2^{-/-}$cells. Cells were transfected with the luciferase reporter in the presence or absence of LGK974 (2 $\mu \mathrm{M})$ and/or exogenously purified WNT-7a (100 nM/ml). Reporter gene activity was determined 24 hours after transfection. (J) Ctnnb1 mRNA levels were also measured in the presence or absence of LGK974 and/ or exogenously purified WNT-7a by quantitative RT-PCR. (K-M) Intracellular calcium sequestration by BAPTA-AM increased Ctnnb1, Wnt7a, and Wnt9a expression. Cells were treated with the Ca ${ }^{2+}$ chelator BAPTA-AM (0.4 $\mu$ M) for 8 hours. Data are presented as mean $\pm \operatorname{SEM}\left({ }^{*} P<0.05,{ }^{* *} P<0.01,{ }^{* * *} P<0.001, n=3\right.$, Student's $t$ test). 
activated Wnt/ $\beta$-catenin activity and increased the Ctnnb1 mRNA levels (Figure 5, I and J), suggesting that Wnt7a upregulation might be the mechanism by which a loss of PC2 increases Wnt/ $\beta$-catenin signaling.

Given that PC2 is a $\mathrm{Ca}^{2+}$-modulated cation channel $(27,75,76)$, we also tested whether chelating intracellular $\mathrm{Ca}^{2+}$ by treating cells with BAPTA-AM could alter the Ctnnb1, Wnt7a, and Wnt9a expression. Ca ${ }^{2+}$ chelation significantly increased the Ctnnb1, Wnt7a, and Wnt9a expression in both WT renal epithelial cells and $P k d 2^{-/-}$cells (Figure 5, K-M), suggesting that PC2 regulates the Ctnnb1, Wnt7a, and Wnt9a expression and subsequent $\beta$-catenin signaling by modulating intracellular $\mathrm{Ca}^{2+}$ concentration.

\section{Discussion}

Previous studies showed that PC2 in association with Wnt signaling induces calcium influx and that the loss of PC2 disturbs polarization during directional cell migration $(27,37,76)$. The present study demonstrated the importance of elevated Wnt/ $\beta$-catenin signaling in the polycystic kidney disease phenotypes caused by a $P k d 2$ loss-of-function mutation in mice. Our results suggested that the loss of $P k d 2$ in renal epithelial cells activates Wnt/ $\beta$-catenin signaling through increasing the autocrine Wnt production (such as $W n t 7 a)$. PC2 appears to regulate the $W n t 7 a$ and $W n t 9 a$ expression by modulating intracellular $\mathrm{Ca}^{2+}$ concentration, while PC2 may regulate Ctnnb1 expression, at least in part, by regulating the $W n t$ gene expression. In renal epithelial cells, Wnt7a not only increased the stability of $\beta$-catenin, but also increased the Ctnnb1 mRNA levels; Wnt inhibitors suppressed this increase. Moreover, downregulating $\beta$-catenin, either genetically or by administering XAV939, which specifically inhibits canonical Wnt signaling, significantly reduced Ctnnb1 expression and improved cystic phenotypes in our ADPKD model. Our findings indicate that canonical Wnt signaling is also involved in the cystogenesis resulting from PC2 deficiency.

We found that BAPTA-AM treatment, which decreases intracellular $\mathrm{Ca}^{2+}$, activated Wnt signaling similarly to $P k d 2^{-/-}$renal epithelial cells. Furthermore, we observed a synergistic effect on the canonical Wnt signaling when $\mathrm{Pkd}^{-1-}$ cells were treated with a $\mathrm{Ca}^{2+}$ chelator. These results suggested that $\mathrm{Ca}^{2+}$ participates in the PC2 deficiency-induced activation of canonical Wnt signaling (27).

Previous studies suggested that the cyst formation in ADPKD can result from excessive fluid secretion into the cyst lumens, aberrant proliferation of the cyst-lining epithelium, and abnormal epithelial polarity $(1,3)$. Drugs, such as vasopressin, triptolide, octreotide, and rapamycin, target the pathways involved in these processes $(65,77-79)$. Although these signaling modulators have shown a reduction in cystogenesis in clinical trials, they do not improve renal function as much as expected $(55,56,58,80-82)$. Of these clinical trials, only tolvaptan has improved renal function more than placebo in ADPKD patients (62), but its clinical application is largely restricted by side effects $(63,64)$. Our present study clearly demonstrated the potential of Wnt/ $\beta$-catenin signaling as a therapeutic target for ADPKD.

We demonstrated that the loss of PC2 upregulated $W n t 7 a$ and $W n t 9 a$ in vitro and in vivo. Wnt7a and $W n t 9 a$ were recently shown to stimulate $\beta$-catenin signaling $(83,84)$. The two small-molecular Wnt inhibitors XAV939 and LGK974 suppressed Wnt/ $\beta$-catenin signaling, significantly extended life span, ameliorated cystic phenotypes, and improved renal functions in our mouse model of $P k d 2$-associated ADPKD (Figure 6 and Supplemental Figure 9). Currently, several Wnt inhibitors are being developed and tested for cancer therapy, including XAV939 and LGK974. It will be interesting to determine whether other Wnt inhibitors are effective treatments for ADPKD models or patients and if antagonizing Wnt can ameliorate ADPKD phenotypes associated with $P k d 1$ mutations or improve other forms of PKD. There is currently no satisfactory clinical therapy for ADPKD. Thus, it is important to test the effectiveness of XAV939 and LGK974 and to identify the PC2/Wnt/ $\beta$-catenin molecular pathway in a mouse ortholog of human ADPKD to assess the potential of these Wnt inhibitors as therapeutic interventions.

\section{Methods}

Mouse models and treatments. All of the mouse models used in this study were from a C57BL/6J inbred background. $P k d 2^{f / \mathrm{f}}$ mice (37) were bred with Villin-Cre-transgenic mice to generate $V i{ }^{\mathrm{Cre}} P k d 2^{\mathrm{f} / \mathrm{f}}$ mice (65). Mice with and without a $P k d 2$ mutation were genotyped by PCR of tail genomic DNA as previously described (37). The primer pairs used for the $P k d 2^{f}$ allele were forward 5'-TCTGACTTGCAGACTGTGGG-3' and reverse 5'-AGGTAGGGGAAGGTCAGGGTTGG-3'. The Vilere transgene was genotyped by amplifying the PCR product with primers against the mouse villin-1 promoter region (forward, 5'-GTGTGGGACAGAGAACAAACCG-3' and reverse, 5'-TGCGAACCTCATCACTCGTTGC-3'). The Ctnnb1 ${ }^{+/-}$mouse line was generated by crossing a Ctnnb1-floxed mouse line ( $C$ tnnb $1^{\mathrm{tm} 2 \mathrm{Kem}}$ or $\left.C \mathrm{tnnb}^{\mathrm{f2}-6}\right)$ from the Jackson Laboratories with a Sox $2^{\text {Cre }}$ mouse line (85). 


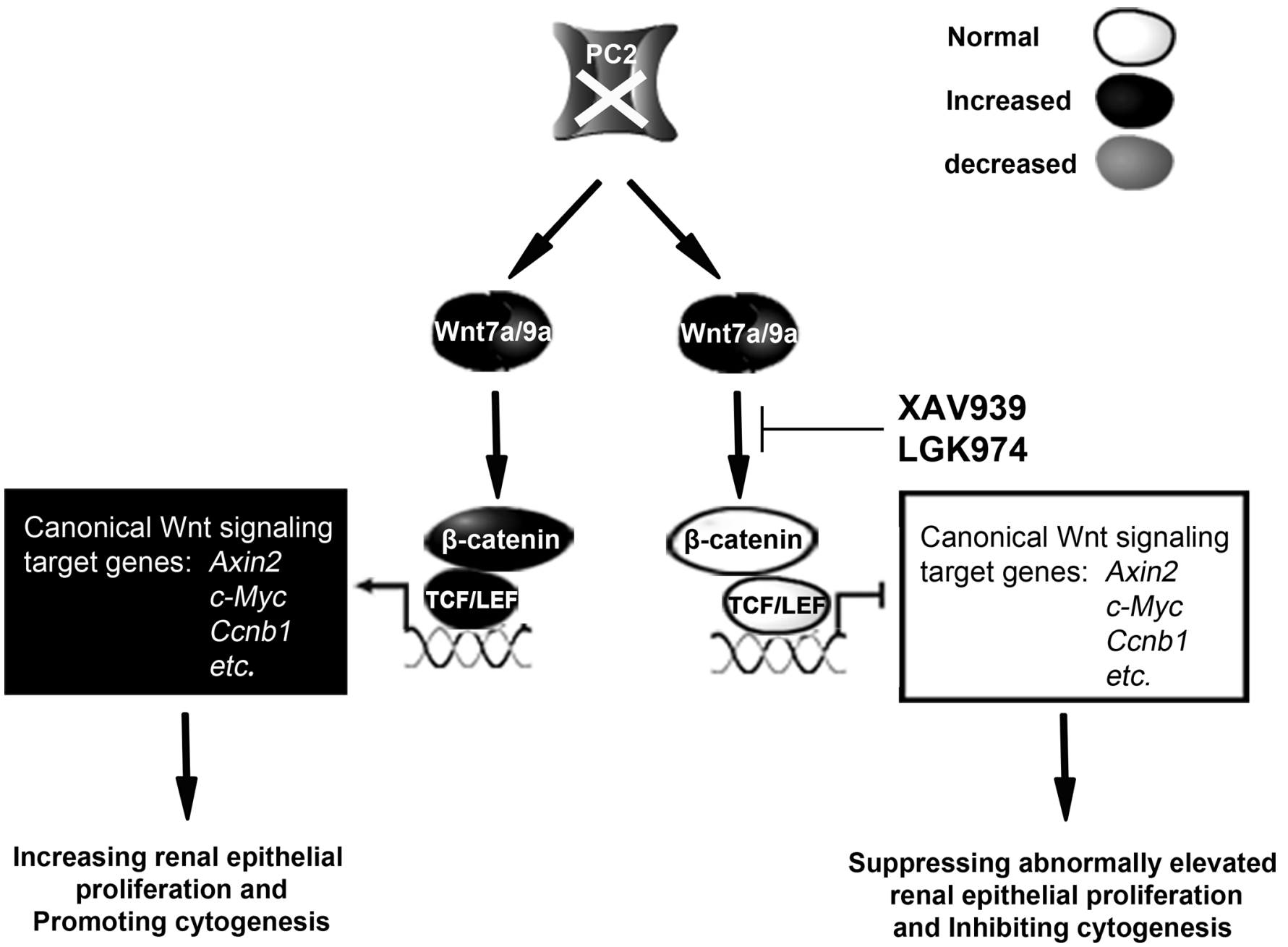

Figure 6. The effect of Wnt inhibitors XAV939 and LGK974 on the PC2/Wnt/ק-catenin molecular pathway. The loss of PC2 elevates Wnt7a and Wnt9a expression, leading to $\beta$-catenin activation and $\beta$-catenin-mediated transcription activation. This eventually increases renal epithelial cell proliferation and promotes cystogenesis. Treatment with XAV939 or LGK974 suppresses $\beta$-catenin activity and decreases the expression of downstream Wnt signaling factors, such as Axin2, c-Myc, and cyclin D1, thereby arresting aberrant cell proliferation and inhibiting cystogenesis.

For XAV939 treatment, male and female $\mathrm{Vil}{ }^{\mathrm{Cre}} \mathrm{Pkd2^{ \textrm {f } / \mathrm { f } }}$ mice were randomly divided into two groups that were given vehicle DMSO (MilliporeSigma) or XAV939 (Selleck) at $50 \mathrm{mg} / \mathrm{kg} / \mathrm{d}$ i.p., using the treatment protocol illustrated in Figure 3A.

For LGK974 treatment, LGK974 was formulated in 10\% (vol/vol) citrate buffer (pH 2.8)/90\% (vol/vol) citrate buffer $(\mathrm{pH} 3.0$ ) or $0.5 \% \mathrm{MC} / 0.5 \%$ Tween 80 and administered by oral gavage at a dosing volume of $10 \mu \mathrm{l} / \mathrm{g}$ animal body weight $(3 \mathrm{mg} / \mathrm{kg} / \mathrm{d})$. Male and female $\mathrm{Vil}{ }^{\mathrm{Cre}} \mathrm{Pkd} 2^{\mathrm{f} / \mathrm{f}}$ mice were randomly divided into two groups that were given vehicle placebo or LGK974 (Selleck) using the treatment protocol illustrated in Figure 4A.

Cell lines and culture. The $P k d 2^{-1-}$ cell line and its maternally derived $P k d 2$ heterozygous $\left(P k d 2^{+-}\right)$cell line were previously described (37). Cell lines were cultured in DMEM/F12 with 10\% FBS. We applied shRNA Lentiviral Transduction Particles SHVRSC-TRCN 0000012691 (MilliporeSigma) to the $P k d 2^{+/-}$and $P k d 2^{-/-}$ cell lines to generate the stable $C$ tnnb1-silenced cell lines $P k d 2^{+--} ; \beta-\mathrm{cat}^{\mathrm{KD}}$ and $P k d 2^{-/-} ; \beta$-cat ${ }^{\mathrm{KD}}$ (Supplemental Figure $4 \mathrm{E}$ ) using an approach similar to that described in our previous study (86). To downregulate Wnt7a expression in $P k d 2^{-/-}$cell lines, the cells were transfected with siRNA specifically targeting mouse Wnt7a or with negative control siRNA (both purchased from Santa Cruz) using the Lipofectamine RNAiMAX Transfection Kit (Invitrogen) according to the manufacturer's instructions. The cells were analyzed by qPCR after 48 hours.

$P K D 2$ was reexpressed in $P k d 2^{-/-}$cells using the pLV-sfGFP(2A) Puro Lentiviral Vector System (Inovogen) with the full-length human $P K D 2$ open reading frame cDNA construct. Recombinant viruses were 
packaged and amplified on a large scale in HEK293 cells; lentiviral particles were purified and used to infect $P k d 2^{-1-}$ cells following the manufacturer's instructions (Addgene).

Antibodies and reagents. The following antibodies, staining materials, and reagents were used: rabbit polyclonal antisera against D682-V968 of the human PC2 COOH-terminus (named hPKD2-Cp), as described in our previous studies (87); anti- $\beta$-actin antibody (catalog A5441), anti- $\alpha$-tubulin antibody (catalog T5201), and DAPI (catalog D9542) (MilliporeSigma); anti-Axin2 antibody (catalog 2151S), anti-cMyc antibody (catalog 13987S), anti-PARP antibody (catalog 9532S), and anti-cleaved caspase-3 (Asp 175) antibody (catalog 9664S) (Cell Signaling Technology); anti-cyclin D1 antibody (catalog sc-70899, Santa Cruz); anti-active- $\beta$-catenin antibody (catalog 05-665, Millipore Corporation); anti- $\beta$-catenin antibody (catalog 610154, BD Biosciences); anti-proliferating cell nuclear antigen (PCNA) (catalog ab92552), anti-Ki67 antibodies (catalog ab58380), and BAPTA-AM (catalog ab120503) (Abcam); and the DeadEnd Fluorometric TUNEL System (catalog G3250, Promega). Secondary antibodies included Cy3-conjugated goat anti-rabbit IgG (catalog AP132C, Millipore Corporation), peroxidase-conjugated rabbit anti-mouse IgG (catalog W4021, Promega), and peroxidase-conjugated goat anti-rabbit IgG (catalog W4011, Promega). Recombinant WNT-7a Protein was purchased from Biolegend (catalog 597902).

Western blots. Fresh tissues or cultured cells were homogenized in RIPA buffer (MilliporeSigma), and protein concentrations were determined by BCA assay (Pierce). Samples were separated by SDS-PAGE and electrotransferred to a nylon membrane (Perkin Elmer). The membrane was incubated with primary antibodies at room temperature for 4 hours and with peroxidase-conjugated secondary antibodies for 1 hour. Antibodies were detected with enhanced chemiluminescence (Pierce). Western blot results were quantified using the densitometry values of the immunoreactive bands for targeted proteins, normalized to loading control. For Western blots, all samples were loaded in duplicate, and all Western blots analyses were repeated at least 3 times. Statistical analysis was based on the results of the duplicated samples of at least 3 experiments.

Histology, immunofluorescence, and Masson trichrome staining. Histology and immunofluorescence staining procedures were as previously described $(37,88,89)$. Masson trichrome staining used a standard protocol. Sections were deparaffinized and rehydrated through 100\% alcohol, 95\% alcohol, and 70\% alcohol and then washed in distilled water. Sections were refixed in Bouin's solution for 1 hour at $56^{\circ} \mathrm{C}$, rinsed with running tap water for 5 minutes, stained in Weigert's iron hematoxylin working solution for 10 minutes, rinsed in running warm tap water for 10 minutes, washed in distilled water, and stained in Biebrich scarlet-acid fuchsin solution for 10 minutes. Sections were incubated with phosphotungstic/phosphomolybdic acid for 10 minutes, after which the solution was discarded and sections were transfered directly into Aniline blue for 5 minutes. Then, sections were rinsed in distilled water, followed by $1 \%$ acetic acid for 1 minute, after which the solution was discarded and sections were rinsed in distilled water, dehydrated, cleared, and coverslipped. For microscopy, we used a Zeiss Axioplan 2IE research microscope and a Zeiss Axiovert 200 inverted microscope system with $\times 10, \times 20$, and $\times 40$ objective lenses. We used an Aperio ScanScope system (Leica) for histological analyses. Digital graphics were viewed and analyzed using ImageScope viewer software version 12.1.0.5029.

Cystic index calculation. We captured 5 representative H\&E images per mouse at $\times 200$ from renal sections from $\mathrm{Vil}^{\mathrm{Cre}} \mathrm{Pkd} 2^{\mathrm{f} / \mathrm{f}}$ mice, $\mathrm{Vil}^{\mathrm{Cre}} \mathrm{Pkd} 2^{\mathrm{f} / \mathrm{f}} \mathrm{Ctnnb1}^{+/-}$mice, placebo-treated or XAV939/LGK974-treated $\mathrm{Vil}$ ${ }^{\mathrm{Cre}} P k d 2^{\mathrm{f} / \mathrm{f}}$ mice, and control $P k d 2^{\mathrm{f} / \mathrm{f}}$ mice. Images were opened in Photoshop software, converted to grayscale, and resized to $800 \times 598$ pixels. A grid was placed over the image to create a total of 1,064 individual points. The points bisecting cyst epithelium and noncystic epithelium were counted, and the percentage of total points that bisected cystic and noncystic epithelium was calculated as the cystic index (45).

Proliferation and apoptosis. To examine cell proliferation, kidneys were fixed in $4 \%$ buffered paraformaldehyde (MilliporeSigma), embedded in paraffin, and sectioned on a microtome. The sections were deparaffinized, rehydrated, and washed in PBS 3 times for 5 minutes each. Epitopes were unmasked by microwave retrieval with citrate buffer ( $\mathrm{pH}$ 6.0). The sections were then immersed in $3 \%$ hydrogen peroxide for 10 minutes to inactivate endogenous peroxidase, washed with PBS 3 times for 5 minutes each, and blocked with 2\% BSA for 1 hour. The sections were then incubated with anti-PCNA and anti-Ki67 antibodies overnight at $4{ }^{\circ} \mathrm{C}$, incubated with a Cy3-conjugated goat anti-rabbit secondary antibody, and stained with DAPI to identify cell nuclei. Proliferation was measured by counting the PCNA- and Ki67-positive nuclei on the cyst-lining epithelia. Nuclei were counted in 3 randomly chosen high-power fields $(\times 20)$ for each sample.

Apoptosis was detected using the DeadEnd Fluorometric TUNEL kit (Promega) and a cleaved caspase-3 antibody according to the manufacturer's instructions. Kidney samples were placed on slides, 
deparaffinized and rehydrated, fixed in 4\% formaldehyde in PBS for 15 minutes, and then permeabilized with Proteinase K (catalog 740506, Macherey Nagel) for 10 minutes. After being washed in PBS, the samples were equilibrated and labeled for 60 minutes. The samples were then immersed in $\times 2$ SSC to stop the reaction and counterstained with DAPI. Positive apoptosis signals were detected by fluorescence microscopy. Apoptotic cells were counted in the same manner as the proliferation markers.

Blood urea nitrogen and creatinine measurements. Following Peking Union Medical College Institutional Animal Care and Use Committee regulations, all mice were anesthetized with isoflurane before cardiac puncture. The blood samples were centrifuged, and the sera were collected. Blood urea nitrogen/creatinine levels were analyzed at the clinical laboratory of Cancer Hospital, Chinese Academy of Medical Sciences.

Microarray analysis, $q P C R$ assays, and pathway analysis. Total RNA was extracted from the WT and Pkd2-/cell lines using Trizol reagent (Invitrogen) according to the manufacturer's instructions. The isolated total RNA samples were purified by the RNeasy Mini Kit (Qiagen), and $50 \mu \mathrm{g}$ total RNA for each sample was sent to the Microarray Core facility at Vanderbilt University. In brief, $10 \mu \mathrm{g}$ labeled cRNA samples were hybridized onto an Affy Mouse MOE430 Array (45,101 different gene probes, Affymetrix) using standard Microarray Core and Affymetrix protocols. Scanned raw data images were processed with GeneChip Operating Software 1.4. Probe set signal intensities were preprocessed and normalized the Vanderbilt Microarray Shared Resource facility. An unsupervised hierarchical cluster with selected proliferation/apoptosis- and Wnt-associated concordant genes was identified using the TIGR MeV program.

For qPCR, total RNA was isolated from the tissues of transgenic and WT mice using Trizol (Invitrogen). The cDNA was generated from the total RNA $(2 \mu \mathrm{g})$ using SuperScript II reverse transcriptase according to the manufacturer's protocol (Invitrogen). The primer pairs used for analysis were (forward) 5'-CACCTCCCAAGTCCTTTATGAATG-3' and (reverse) 5'-AGCGTCAAACTGCGTGGATG-3' for mouse Ctnnb1, (forward) 5'-CCTGGACGAGTGTCAGTTTCA-3' and (reverse) 5'-ATCGCATAGGTGAAGGCAGC-3' for mouse Wnt7a, (forward) 5'-GACAACCTCAAGTACAGCAG-3' and (reverse) 5'-TTCCACTCCAGCCTTTATCAC-3' for mouse Wnt9a, and (forward) 5'-GACCACAGTCCATGCCATCAC-3' and (reverse) 5'-TCCACCACCCTGTTGCTGTA-3' for GAPDH as an mRNA loading control. The PCR products were separated by $1.5 \%$ agarose gel electrophoresis. Quantitative PCR was performed using the iCycler iQ RealTime PCR Detection System with the iQ SYBR Green Supermix kit (Bio-Rad).

For pathway analysis, gene set enrichment analysis (GSEA) (http://software.broadinstitute.org/gsea/ msigdb) was used to infer global network functions of all differentially expressed genes between WT and Pkd2 ${ }^{-/-}$cells. GenBank Accession numbers (NM_013630, BB249222, AI182499, AK004683, AB072311, AB073819, NM_007614, NM_007631, BC006728, AV213413, AK004781, AI323642, BB086994, BB067079, BB398993) and fold change in expression (> \pm 4 ) of the genes were uploaded into the GSEA to group the interaction networks and the biological functions of differentially expressed genes. The significance ( $P$ value of overlap) was calculated by Fisher's exact test.

Luciferase reporter assay. $P k d 2^{+/-}$and $P k d 2^{-/-}$cells (37) grown in 24-well culture plates were transiently transfected with the LEF reporter plasmid (30 ng), LEF plasmid (30 ng), LacZ plasmid (160 ng), and GFP plasmid (30 ng). DNA ( $1 \mu \mathrm{g}$ ) was added to each well, and the cells were maintained in serum-containing medium. Luciferase activity was determined using a luciferase assay system (Promega) and luminometer according to the manufacturer's specifications. Luciferase activity with LEF reporter was normalized to GFP for each cell line and analyzed statistically.

TOPflash luciferase reporter assay. $\mathrm{Pkd} 2^{+/-}$and $\mathrm{Pkd}^{-/-}$cells (37) grown in 12-well culture plates were transiently transfected with either the TOPflash TCF reporter plasmid $(0.2 \mathrm{mg})$ or the FOPflash reporter plasmid $(0.2 \mathrm{mg})$ bearing a mutated TCF/LEF-binding site. DNA $(1 \mu \mathrm{g})$ was added to each well, and the cells were maintained in serum-containing medium. Cells were harvested 24 hours after transfection in $100-\mu 1$ cell lysis buffer (Promega). Luciferase activity was determined using a luciferase assay system (Promega) and luminometer according to the manufacturer's specifications. Luciferase activity with TOPflash was normalized by FOPflash for each cell line and analyzed statistically.

Statistics. Minimal group sizes were determined by power calculations using the DSS Researcher's Toolkit with an $\alpha$ of 0.05 and a power of 0.8 . Animals were grouped unblinded but were randomized, and investigators were blinded for most of the qualification experiments. No samples or animals were excluded from analysis. Assumptions concerning the data, including normal distribution and similar variation between experimental groups, were examined for appropriateness before conducting statistical tests. Comparisons between two groups were performed by unpaired 2-tailed $t$ test, and comparisons between more 
than two groups were performed by 1-way ANOVA, followed by Bonferroni's post-hoc test using Prism 6.0 software (GraphPad). Statistical tests used biological replicates. A $P$ value of less than 0.05 was considered statistically significant.

Study approval. All animal experiments were performed according to protocols approved by the Institutional Animal Care and Use Committee of Peking Union Medical College.

\section{Author contributions}

GW, DW, and CL conceived and designed the experiments. AL, YL, YX, SF, JM, and XS performed the experiments. AL, YX, SF, JM, XS, LZ, XZ, QX, DW, and GW analyzed and interpreted the data. AL, GW, and DW wrote the manuscript.

\section{Acknowledgments}

This work was supported by the National Natural Science Foundation of China $(81173114,81072688$, 81473282, and 81673402 to GW), Sci-Tech Planning Projects of Anhui Province (1704e1002230 to CL), and by an NIH grant (GM112182 to DW).

Address correspondence to: Dianqing Wu, Department of Pharmacology, Yale University School of Medicine, New Haven, Connecticut 06520, USA. Phone: 203.785.3149; Email: dianqing.wu@yale.edu. Or to: Guanqing Wu or Chaozhao Liang, Anhui Province PKD Center, Institute/Department of Urology, The First Affiliated Hospital of Anhui Medical University, Hefei, Anhui Province, 230022, China. Phone: 86.551.62923865; Email: guanqing.wu@ahmu.edu.cn(G. Wu). Phone: 86.551.62923856; Email: liang_chaozhao@ahmu.edu.cn (C. Liang).

1. Grantham JJ, Torres VE. The importance of total kidney volume in evaluating progression of polycystic kidney disease. Nat Rev Nephrol. 2016;12(11):667-677.

2. Gallagher AR, Germino GG, Somlo S. Molecular advances in autosomal dominant polycystic kidney disease. Adv Chronic Kidney Dis. 2010;17(2):118-130.

3. Harris PC, Torres VE. Genetic mechanisms and signaling pathways in autosomal dominant polycystic kidney disease. JClin Invest. 2014;124(6):2315-2324.

4. Ong AC, Devuyst O, Knebelmann B, Walz G, ERA-EDTA Working Group for Inherited Kidney Diseases. Autosomal dominant polycystic kidney disease: the changing face of clinical management. Lancet. 2015;385(9981):1993-2002.

5. Fedeles SV, Gallagher AR, Somlo S. Polycystin-1: a master regulator of intersecting cystic pathways. Trends Mol Med. 2014;20(5):251-260

6. Igarashi P, Somlo S. Polycystic kidney disease. J Am Soc Nephrol. 2007;18(5):1371-1373.

7. Spirli C, et al. Mammalian target of rapamycin regulates vascular endothelial growth factor-dependent liver cyst growth in polycystin-2-defective mice. Hepatology. 2010;51(5):1778-1788.

8. Bae KT, et al. Magnetic resonance imaging evaluation of hepatic cysts in early autosomal-dominant polycystic kidney disease: the Consortium for Radiologic Imaging Studies of Polycystic Kidney Disease cohort. Clin J Am Soc Nephrol. 2006;1(1):64-69.

9. Bae KT, Grantham JJ. Imaging for the prognosis of autosomal dominant polycystic kidney disease. Nat Rev Nephrol. 2010;6(2):96-106

10. Torra R, et al. Ultrasonographic study of pancreatic cysts in autosomal dominant polycystic kidney disease. Clin Nephrol. 1997;47(1):19-22.

11. Yazdanpanah K, Manouchehri N, Hosseinzadeh E, Emami MH, Karami M, Sarrami AH. Recurrent acute pancreatitis and cholangitis in a patient with autosomal dominant polycystic kidney disease. Int J Prev Med. 2013;4(2):233-236.

12. Ring T, Spiegelhalter D. Risk of intracranial aneurysm bleeding in autosomal-dominant polycystic kidney disease. Kidney Int. 2007;72(11):1400-1402.

13. Ong AC. Screening for intracranial aneurysms in ADPKD. BMJ. 2009;339:b3763.

14. Xu HW, Yu SQ, Mei CL, Li MH. Screening for intracranial aneurysm in 355 patients with autosomal-dominant polycystic kidney disease. Stroke. 2011;42(1):204-206.

15. Rozenfeld MN, Ansari SA, Mohan P, Shaibani A, Russell EJ, Hurley MC. Autosomal Dominant Polycystic Kidney Disease and Intracranial Aneurysms: Is There an Increased Risk of Treatment? AJNR Am J Neuroradiol. 2016;37(2):290-293.

16. Adeola T, Adeleye O, Potts JL, Faulkner M, Oso A. Thoracic aortic dissection in a patient with autosomal dominant polycystic kidney disease. J Natl Med Assoc. 2001;93(7-8):282-287.

17. Liu D, et al. A Pkd1-Fbn1 genetic interaction implicates TGF- $\beta$ signaling in the pathogenesis of vascular complications in autosomal dominant polycystic kidney disease. J Am Soc Nephrol. 2014;25(1):81-91.

18. Perrone RD, Malek AM, Watnick T. Vascular complications in autosomal dominant polycystic kidney disease. Nat Rev Nephrol. 2015;11(10):589-598.

19. Ong AC, Harris PC. A polycystin-centric view of cyst formation and disease: the polycystins revisited. Kidney Int. 2015;88(4):699-710.

20. Germino GG. Linking cilia to Wnts. Nat Genet. 2005;37(5):455-457.

21. Benzing T, Simons M, Walz G. Wnt signaling in polycystic kidney disease. J Am Soc Nephrol. 2007;18(5):1389-1398. 
22. Song X, et al. Systems biology of autosomal dominant polycystic kidney disease (ADPKD): computational identification of gene expression pathways and integrated regulatory networks. Hum Mol Genet. 2009;18(13):2328-2343.

23. Lancaster MA, Gleeson JG. Cystic kidney disease: the role of Wnt signaling. Trends Mol Med. 2010;16(8):349-360.

24. Patel V. Balancing the Wnts in polycystic kidney disease. J Am Soc Nephrol. 2010;21(9):1412-1414.

25. Cnossen WR, et al. LRP5 variants may contribute to ADPKD. Eur J Hum Genet. 2016;24(2):237-242.

26. Feng $\mathrm{S}$, et al. The sorting nexin 3 retromer pathway regulates the cell surface localization and activity of a Wnt-activated polycystin channel complex. J Am Soc Nephrol. 2017;28(10):2973-2984.

27. Kim S, et al. The polycystin complex mediates Wnt/Ca(2+) signalling. Nat Cell Biol. 2016;18(7):752-764.

28. Sugiyama N, Tsukiyama T, Yamaguchi TP, Yokoyama T. The canonical Wnt signaling pathway is not involved in renal cyst development in the kidneys of inv mutant mice. Kidney Int. 2011;79(9):957-965.

29. Luyten A, et al. Aberrant regulation of planar cell polarity in polycystic kidney disease. J Am Soc Nephrol. 2010;21(9):1521-1532.

30. Happé $\mathrm{H}$, et al. Toxic tubular injury in kidneys from Pkd1-deletion mice accelerates cystogenesis accompanied by dysregulated planar cell polarity and canonical Wnt signaling pathways. Hum Mol Genet. 2009;18(14):2532-2542.

31. Karner CM, Chirumamilla R, Aoki S, Igarashi P, Wallingford JB, Carroll TJ. Wnt9b signaling regulates planar cell polarity and kidney tubule morphogenesis. Nat Genet. 2009;41(7):793-799.

32. Fischer E, et al. Defective planar cell polarity in polycystic kidney disease. Nat Genet. 2006;38(1):21-23.

33. Saadi-Kheddouci S, et al. Early development of polycystic kidney disease in transgenic mice expressing an activated mutant of the beta-catenin gene. Oncogene. 2001;20(42):5972-5981.

34. Qian CN, et al. Cystic renal neoplasia following conditional inactivation of apc in mouse renal tubular epithelium. J Biol Chem. 2005;280(5):3938-3945.

35. Trudel M, D'Agati V, Costantini F. C-myc as an inducer of polycystic kidney disease in transgenic mice. Kidney Int. 1991;39(4):665-671.

36. Lal M, et al. Polycystin-1 C-terminal tail associates with beta-catenin and inhibits canonical Wnt signaling. Hum Mol Genet. 2008;17(20):3105-3117.

37. Kim I, et al. Conditional mutation of Pkd2 causes cystogenesis and upregulates beta-catenin. J Am Soc Nephrol. 2009;20(12):2556-2569.

38. Merrick D, et al. The $\gamma$-secretase cleavage product of polycystin-1 regulates TCF and CHOP-mediated transcriptional activation through a p300-dependent mechanism. Dev Cell. 2012;22(1):197-210.

39. Marose TD, Merkel CE, McMahon AP, Carroll TJ. Beta-catenin is necessary to keep cells of ureteric bud/Wolffian duct epithelium in a precursor state. Dev Biol. 2008;314(1):112-126.

40. Miller MM, et al. T-cell factor/ $\beta$-catenin activity is suppressed in two different models of autosomal dominant polycystic kidney disease. Kidney Int. 2011;80(2):146-153.

41. Qin S, Taglienti M, Cai L, Zhou J, Kreidberg JA. c-Met and NF- $\kappa$ B-dependent overexpression of Wnt7a and -7b and Pax2 promotes cystogenesis in polycystic kidney disease. J Am Soc Nephrol. 2012;23(8):1309-1318.

42. Lancaster MA, et al. Impaired Wnt-beta-catenin signaling disrupts adult renal homeostasis and leads to cystic kidney ciliopathy. Nat Med. 2009;15(9):1046-1054.

43. Wuebken A, Schmidt-Ott KM. WNT/ $\beta$-catenin signaling in polycystic kidney disease. Kidney Int. 2011;80(2):135-138

44. Zafar I, Ravichandran K, Belibi FA, Doctor RB, Edelstein CL. Sirolimus attenuates disease progression in an orthologous mouse model of human autosomal dominant polycystic kidney disease. Kidney Int. 2010;78(8):754-761.

45. Shillingford JM, Piontek KB, Germino GG, Weimbs T. Rapamycin ameliorates PKD resulting from conditional inactivation of Pkd1. J Am Soc Nephrol. 2010;21(3):489-497.

46. Belibi F, Ravichandran K, Zafar I, He Z, Edelstein CL. mTORC1/2 and rapamycin in female Han:SPRD rats with polycystic kidney disease. Am J Physiol Renal Physiol. 2011;300(1):F236-F244.

47. Stayner C, Shields J, Slobbe L, Shillingford JM, Weimbs T, Eccles MR. Rapamycin-mediated suppression of renal cyst expansion in del34 Pkd1-/- mutant mouse embryos: an investigation of the feasibility of renal cyst prevention in the foetus. Nephrology (Carlton). 2012;17(8):739-747.

48. Shillingford JM, Leamon CP, Vlahov IR, Weimbs T. Folate-conjugated rapamycin slows progression of polycystic kidney disease. J Am Soc Nephrol. 2012;23(10):1674-1681.

49. Novalic Z, et al. Dose-dependent effects of sirolimus on mTOR signaling and polycystic kidney disease. J Am Soc Nephrol. 2012;23(5):842-853.

50. Sabbatini M, et al. Effects of combined administration of rapamycin, tolvaptan, and AEZ-131 on the progression of polycystic disease in PCK rats. Am J Physiol Renal Physiol. 2014;306(10):F1243-F1250.

51. Ravichandran K, Zafar I, Ozkok A, Edelstein CL. An mTOR kinase inhibitor slows disease progression in a rat model of polycystic kidney disease. Nephrol Dial Transplant. 2015;30(1):45-53.

52. Soliman A, Zamil S, Lotfy A, Ismail E. Sirolimus produced S-shaped effect on adult polycystic kidneys after 2-year treatment. Transplant Proc. 2012;44(10):2936-2939.

53. Leonhard WN, et al. Scattered deletion of PKD1 in kidneys causes a cystic snowball effect and recapitulates polycystic kidney disease. J Am Soc Nephrol. 2015;26(6):1322-1333.

54. Torres VE, et al. Multicenter, open-label, extension trial to evaluate the long-term efficacy and safety of early versus delayed treatment with tolvaptan in autosomal dominant polycystic kidney disease: the TEMPO 4:4 Trial. Nephrol Dial Transplant. 2017;32(7):1262.

55. Serra AL, et al. Sirolimus and kidney growth in autosomal dominant polycystic kidney disease. $N$ Engl J Med. 2010;363(9):820-829.

56. Walz G, et al. Everolimus in patients with autosomal dominant polycystic kidney disease. NEngl J Med. 2010;363(9):830-840.

57. Ruggenenti $\mathrm{P}$, et al. Safety and efficacy of long-acting somatostatin treatment in autosomal-dominant polycystic kidney disease. Kidney Int. 2005;68(1):206-216.

58. Caroli A, et al. Effect of longacting somatostatin analogue on kidney and cyst growth in autosomal dominant polycystic kidney 
disease (ALADIN): a randomised, placebo-controlled, multicentre trial. Lancet. 2013;382(9903):1485-1495.

59. Torres VE, et al. Angiotensin blockade in late autosomal dominant polycystic kidney disease. $N$ Engl J Med. 2014;371(24):2267-2276.

60. Schrier RW, et al. Blood pressure in early autosomal dominant polycystic kidney disease. $N$ Engl J Med. 2014;371(24):2255-2266.

61. Tesar V, et al. Bosutinib versus placebo for autosomal dominant polycystic kidney disease. J Am Soc Nephrol. 2017;28(11):3404-3413.

62. Torres VE, et al. Tolvaptan in patients with autosomal dominant polycystic kidney disease. N Engl J Med. 2012;367(25):2407-2418

63. Blair HA, Keating GM. Tolvaptan: A review in autosomal dominant polycystic kidney disease. Drugs. 2015;75(15):1797-1806.

64. Horie S. Will introduction of tolvaptan change clinical practice in autosomal dominant polycystic kidney disease? Kidney Int. 2015;88(1):14-16

65. Li A, et al. Rapamycin treatment dose-dependently improves the cystic kidney in a new ADPKD mouse model via the mTORC1 and cell-cycle-associated CDK1/cyclin axis. J Cell Mol Med. 2017;21(8):1619-1635.

66. Chapin HC, Caplan MJ. The cell biology of polycystic kidney disease. J Cell Biol. 2010;191(4):701-710.

67. Clevers H, Nusse R. Wnt/ $\beta$-catenin signaling and disease. Cell. 2012;149(6):1192-1205.

68. Polakis P. Wnt signaling in cancer. Cold Spring Harb Perspect Biol. 2012;4(5):a008052.

69. MacDonald BT, Tamai K, He X. Wnt/beta-catenin signaling: components, mechanisms, and diseases. Dev Cell. 2009;17(1):9-26.

70. Niehrs C. The complex world of WNT receptor signalling. Nat Rev Mol Cell Biol. 2012;13(12):767-779.

71. Huang SM, et al. Tankyrase inhibition stabilizes axin and antagonizes Wnt signalling. Nature. 2009;461(7264):614-620.

72. Jiang X, et al. Inactivating mutations of RNF43 confer Wnt dependency in pancreatic ductal adenocarcinoma. Proc Natl Acad Sci USA. 2013;110(31):12649-12654.

73. Liu J, et al. Targeting Wnt-driven cancer through the inhibition of Porcupine by LGK974. Proc Natl Acad Sci USA 2013;110(50):20224-20229.

74. Hu B, et al. Cystogenesis in ARPKD results from increased apoptosis in collecting duct epithelial cells of Pkhd1 mutant kidneys. Exp Cell Res. 2011;317(2):173-187.

75. Mochizuki T, et al. PKD2, a gene for polycystic kidney disease that encodes an integral membrane protein. Science. 1996;272(5266):1339-1342.

76. Koulen P, et al. Polycystin-2 is an intracellular calcium release channel. Nat Cell Biol. 2002;4(3):191-197.

77. Torres VE. Vasopressin antagonists in polycystic kidney disease. Kidney Int. 2005;68(5):2405-2418.

78. Leuenroth SJ, Bencivenga N, Igarashi P, Somlo S, Crews CM. Triptolide reduces cystogenesis in a model of ADPKD. J Am Soc Nephrol. 2008;19(9):1659-1662.

79. Masyuk TV, et al. Pasireotide is more effective than octreotide in reducing hepatorenal cystogenesis in rodents with polycystic kidney and liver diseases. Hepatology. 2013;58(1):409-421

80. Barash I, Ponda MP, Goldfarb DS, Skolnik EY. A pilot clinical study to evaluate changes in urine osmolality and urine cAMP in response to acute and chronic water loading in autosomal dominant polycystic kidney disease. Clin J Am Soc Nephrol. 2010;5(4):693-697.

81. Calvet JP. MEK inhibition holds promise for polycystic kidney disease. J Am Soc Nephrol. 2006;17(6):1498-1500

82. Perico N, et al. Sirolimus therapy to halt the progression of ADPKD. J Am Soc Nephrol. 2010;21(6):1031-1040.

83. Green JL, et al. Use of a molecular genetic platform technology to produce human Wnt proteins reveals distinct local and distal signaling abilities. PLoS One. 2013;8(3):e58395.

84. DiRenzo DM, et al. A crosstalk between TGF- $\beta /$ Smad3 and Wnt/ $\beta$-catenin pathways promotes vascular smooth muscle cell proliferation. Cell Signal. 2016;28(5):498-505.

85. Hayashi S, Lewis P, Pevny L, McMahon AP. Efficient gene modulation in mouse epiblast using a Sox2Cre transgenic mouse strain. Mech Dev. 2002;119 Suppl 1:S97-S101.

86. Mai W, et al. Inhibition of Pkhd1 impairs tubulomorphogenesis of cultured IMCD cells. Mol Biol Cell. 2005;16(9):4398-4409.

87. Kim I, et al. Fibrocystin/polyductin modulates renal tubular formation by regulating polycystin-2 expression and function. $J$ Am Soc Nephrol. 2008;19(3):455-468.

88. Li A, et al. Human polycystin-2 transgene dose-dependently rescues ADPKD phenotypes in Pkd2 mutant mice. Am J Pathol. 2015;185(10):2843-2860.

89. Zhang MZ, et al. PKHD1 protein encoded by the gene for autosomal recessive polycystic kidney disease associates with basal bodies and primary cilia in renal epithelial cells. Proc Natl Acad Sci USA. 2004;101(8):2311-2316. 